\title{
NEUROKUTANE ERKRANKUNGEN: ALTBEKANNT UND NEU ENTDECKT
}

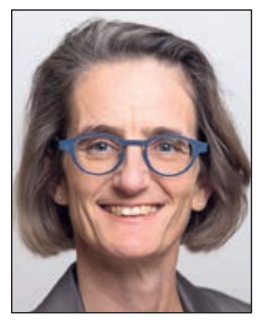

Sandra Tölle

https://doi.org/ 10.35190/d2021.3.4

\section{Kurze Einführung}

Die Assoziation von Entwicklungsanomalien von Hirn, Haut und Augen wurde 1932 vom Niederländer Ophthalmologen Jan van der Hoeve ${ }^{1)}$ unter dem Begriff Phakomatosen zusammengefasst. Er gruppierte darunter die Neurofibromatose, die Tuberöse Sklerose und später die von Hippel-Lindau Erkrankung. Zeitgleich schlugen Paul Ivan Yakovlev und Riley H. Guthrie auf der anderen Seite des Atlantiks den Begriff Neurokutane Erkrankungen vor, geprägt durch ihre Erkenntnisse, dass kongenitale Fehlbildungen des Ektoderms (Haut, Hirn, Retina, Auge) wie bei der Neurofibromatose, der tuberösen Sklerose oder dem Sturge-Weber-Syndrom klinisch häufig die Gemeinsamkeiten von Hautmanifestationen, kognitiver Beeinträchtigung und Epilepsie aufwiesen. Sie erkannten auch, dass die Haut ein diagnostisches Fenster zum zentralen Nervensystem ist, was auch heute noch zutrifft²).

Beide Begriffe wurden im Verlauf der Jahrzehnte synonym verwendet. Heute versteht man darunter eine grosse heterogene Gruppe von Erkrankungen, die Haut, Auge und zentrales und peripheres Nervensystem betreffen, wobei auch andere Organe (z.B. Herz, Lungen, Nieren, Blutgefässe und Knochen) mitbeteiligt sein können ${ }^{3}$. Die beschriebenen Erkrankungen kommen relativ selten vor, aber in der Summe und der häufig doch schweren Auswirkungen schon im Kindesalter bilden sie einen relevanten Anteil im neuropädiatrischen Kollektiv.

Nach der Erstbeschreibung der «klassischen» Phakomatosen wie der Neurofibromatose und der Tuberösen Sklerose Ende des 19. Jahrhunderts, erfolgte Ende des 20. Jahrhunderts die genetische Zuordnung und in den letzten Jahrzehnten dank grosser Fortschritte in der Molekulargenetik, die Analyse der zellulären Basis, der intra- und extraneuronalen Signalwege. Dies ermöglichte ein besseres Verständnis der zugrundeliegenden Pathogenese und letztlich die Entwicklung von neuen zielgerichteten Therapien. Im Folgenden soll am Beispiel von drei neurokutanen Krankheiten auf die neueren Therapiemöglichkeiten eingegangen werden.

\section{Neurofibromatose Typ 1 (NF1)}

Mit einer Häufigkeit von ca. 1:3000 ist die NF1 die häu-
Eine inaktivierende Mutation in NF1, einem Tumor Suppressor Gen, führt zur Fehlfunktion des RAS Inhibitors Neurofibromin und durch gestörte Regulation von Zellwachstum ${ }^{4)}$ zu einer Häufung von benignen und malignen Tumoren. NF1 kann fast jedes Organsystem betreffen mit einer breiten Palette an Erscheinungsformen. Die klassische Präsentation ist ein sich motorisch langsam entwickelndes Kleinkind mit muskulärer Hypotonie, leichter Trichterbrust, relativer Makrozephalie und Café au lait Flecken. Rund $60 \%$ der Kinder leiden im Verlauf unter schulischen Leistungsproblemen und Aufmerksamkeitsdefiziten. Deutlich seltener treten Tumoren des zentralen Nervensystems, plexiforme Neurofibrome, Knochenanomalien (Verbiegung der langen Röhrenknochen, Skoliose) oder Vaskulopathien (Moyamoya Syndrom) auf. Die Diagnose der NF1 kann klinisch gestellt werden (vgl. Tabelle 1 und Abbildung 1), wobei mit Anwendung der revidierten Diagnosekriterien neu auch der genetische Nachweis einer pathogenen NF1 Variante zählt5).

\section{Sehbahntumoren (optic pathway glioma)} Ca. 15-20\% der Kinder mit NF1 entwickeln einen Sehbahntumor, wobei nur ca. 35\% der Sehbahntumoren progredient wachsen, Symptome verursachen und eine Behandlung erfordern. NF1 assoziierte Sehbahngliome werden am häufigsten bei Kindern unter 7 Jahren (Mittelwert 4,5 Jahre) gesehen ${ }^{6}$. Die Sehbahngliome können entlang der gesamten Sehbahn auftreten und lokalisationsabhängig unterschiedliche Symptome hervorrufen. Klinische Zeichen eines Sehbahnglioms können eine Visusminderung, eine Gesichtsfeldeinschränkung, ein Strabismus, ein relatives Afferenzdefizit, ein Papillenödem oder eine Protrusio bulbi sein. Bei Chiasma- und Hypothalamusbeteiligung können auch endokrine Auffälligkeiten wie vorzeitige Pubertätszeichen oder ein Wachstumshormonmangel gesehen werden bis hin zum sehr seltenen Masseneffekt, dienzephalen Syndrom oder obstruktiven Hydrozephalus. Regelmässige ophthalmologische Kontrollen sind dementsprechend wichtig und werden halbjährlich bis jährlich bis zum Alter von sechs Jahren, jährlich bis zum Alter von mindestens zehn Jahren und alle zwei Jahre bis zum Alter von 18 Jahren empfohlen ${ }^{6,7)}$. MRI Untersuchungen als Screening sind nicht indiziert, zumal die zufällige Entdeckung eines Sehbahntumors bei fehlenden Symptomen nur selten einen Einfluss hat auf das Management des Patien$\operatorname{ten}^{8}$. 


\section{Fortbildung}

A Mindestens 2 der folgenden Kriterien müssen erfüllt sein bei einem Kind ohne Elternteil mit NF1

- $\geq 6$ Café au lait Flecken $\geq 5 \mathrm{~mm}$ präpuberal bzw. $\geq 15 \mathrm{~mm}$ postpuberal

- Freckling (vor allem axillär und/oder inguinal)

- $\geq 2$ Neurofibrome oder ein plexiformes Neurofibrom

- Sehbahngliom

- $\geq 2$ Irishamartome (Lisch-Knötchen) oder $\geq 2$ Choroidale Anomalien

- Typische Knochenveränderungen (Sphenoiddysplasie, anterolaterale Verbiegung der Tibia oder Pseudarthrose eines langen Röhrenknochens)

- Heterozygote pathogene NF1 Variante mit einer Varianten Allelfrequenz von 50\% in scheinbar normalem Gewebe wie Leukozyten

B Mindestens 1 der obigen Kriterien muss erfüllt sein, wenn das Kind einen Elternteil hat mit NF1 gemäss den unter A gelisteten Kriterien

Tabelle 1: Redividierte diagnostische Kriterien für Neurofibromatose Typ 1

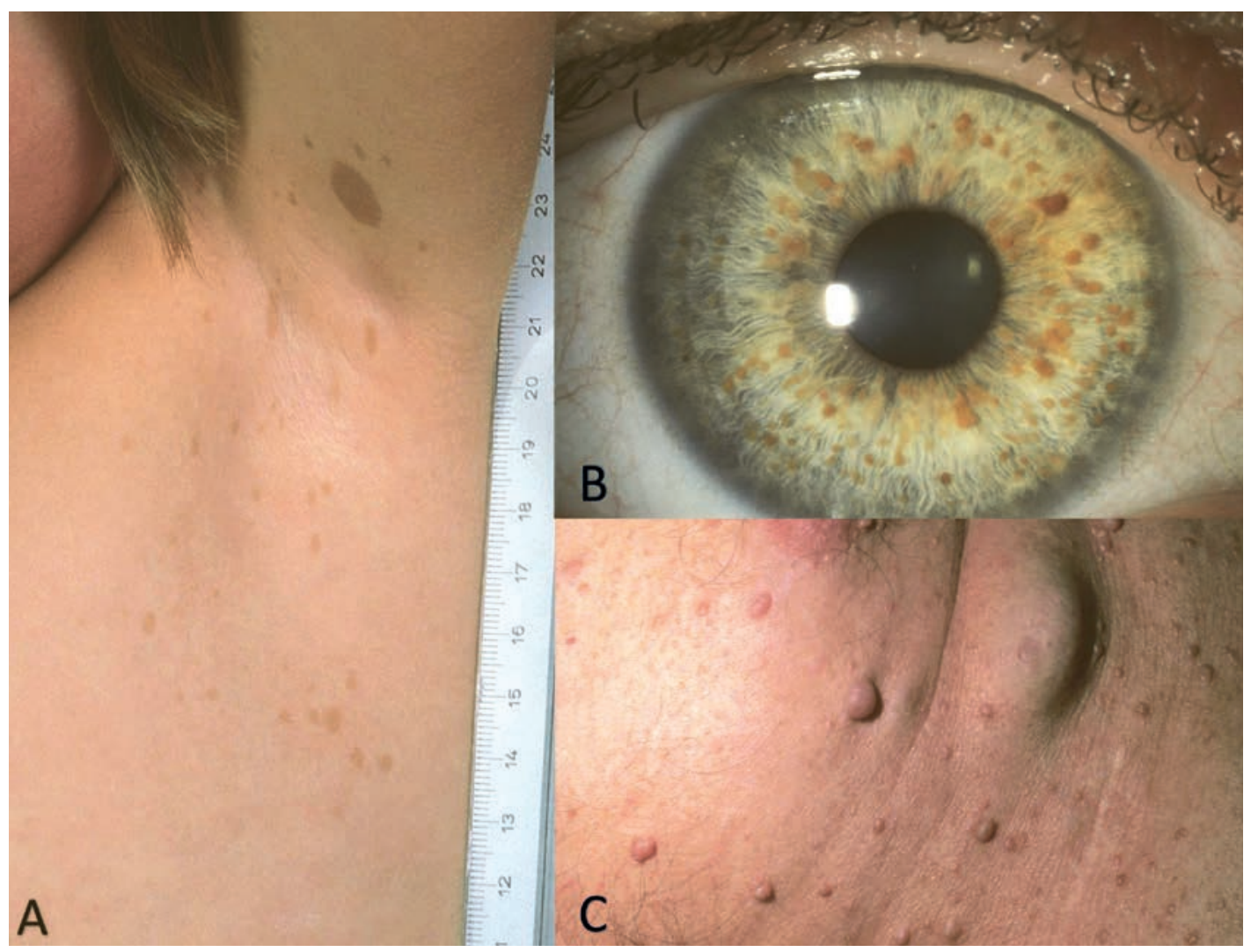

Abbildung 1: Fotos bei Neurofibromatose Typ 1

A Café au lait Fleck und axilläres Freckling

$B$ Lisch Knötchen auf blau pigmentierter Iris

C Kutane Neurofibrome beim Erwachsenen

\section{Behandlung}

Die heutigen Guidelines empfehlen eine Intervention nur, wenn es eine dokumentierte Verschlechterung des Sehens oder einen radiologischen Progress gibt ${ }^{6}$ ). Eine Chemotherapie mit Vincristin und Carboplatin oder nur Vinblastin wird an vielen Kliniken als erster Behandlungsschritt empfohlen mit der Aussicht auf Langzeittumorkontrolle. Bezogen auf die Sehleistung wurde von Fisher et al. ${ }^{9}$ in einer Studie mit 115 NF1
Patienten mit Sehbahntumor und Chemotherapie gezeigt, dass 32\% eine Verbesserung erzielten, 40\% eine Stabilisierung und $28 \%$ eine Verschlechterung. Andere Therapiemodalitäten, die üblicherweise bei Hirntumoren erwogen werden, sind problematisch: Die Neurochirurgie ist aufgrund der Lokalisation limitiert und die Bestrahlung wird bei Kindern mit NF1 wegen dem Risiko von Zweittumoren und Moyamoya Syndrom nicht empfohlen ${ }^{10}$. Die Anwendung gezielter bio- 


\section{Fortbildung}

logischer Therapien bekommt mit der Entschlüsselung der zellulären Pathways einen zunehmenden Stellenwert. Neuere Daten aus präklinischen Modellen haben die Überaktivierung des MAPK/ERK/MEK Pathway als direkte Konsequenz des NF1 assoziierten Funktionsverlustes von Neurofibromin bestätigt ${ }^{11)}$. In der Folge wurden Studien mit Inhibitoren des Mitogen-activated protein kinase (MAPK) Weges durchgeführt. Eine kürzlich publizierte Phase-II-Studie mit dem MEK-Inhibitor Selumetinib hat bei Patienten mit Rezidiven von Low grade Gliomen (inkl. Sehbahntumoren) in 40\% der Fälle ein klares Ansprechen gezeigt ${ }^{12}$. Nebst Selumetinib sind weitere MEK-Inhibitoren in Studien in Erprobung. In einer Phase-II-Studie mit Kindern und NF1 assoziierten low grade Gliomen hat der mTOR-Inhibitor Everolimus ebenfalls gute Resultate gezeigt ${ }^{13)}$.

\section{Plexiforme Neurofibrome}

Die plexiformen Neurofibrome sind angeborene Tumoren, die sich netzartig entlang peripherer Nerven ausbreiten und keine Wachstumsgrenzen respektieren. Sie führen dadurch zu Funktionseinbussen, Schmerzen, Entstellung mit verringerter Lebensqualität und bei entsprechender Lokalisation auch zur Verlegung von Atemwegen. Gross et al. ${ }^{14)}$ haben 2020 in einer Phase-II-Studie mit 50 Kindern und inoperablen plexiformen Neurofibromen gezeigt, dass mit dem MEK-Inhibitor Selumetinib in der Mehrheit der Patienten eine Tumorvolumenreduktion resultierte mit klinisch relevantem Benefit z.B. hinsichtlich der Schmerzen. Das Schrumpfen der Tumore und das fehlende Tumorwachstum unter dieser Therapie steht in Kontrast zu den Befunden der National Cancer Institute
(NCl) natural-history study, bei welchen die meisten Patienten einen Progress des Tumors zeigten.

\section{Tuberöse Sklerose Complex (TSC)}

Die TSC ist eine Multisystemerkrankung mit sehr unterschiedlicher Ausprägung. Die Häufigkeit der autosomal dominanten Erkrankung wird auf ca. 1:6000 -1:10000 geschätzt. Die Mehrheit der Fälle weist eine Neumutation auf: Im TSC1 (auf Chromosom 9q34) oder TSC2 (auf Chromosom 16p13) Gen. Die jeweiligen Genprodukte Hamartin bzw. Tuberin sind wichtige Regulatoren des mTORC1-Pathway (mammalian Target of Rapamycin). Die TSC Mutation führt zur abnormen Aktivierung dieses mTORC1 Pathway und so zu unkontrollierter Zellproliferation und Wachstum von Tumoren in vielen Organsystemen. Für die Diagnose ausreichend ist der isolierte Nachweis einer pathogenen Mutation im TSC1 oder TSC2. Im klinischen Alltag sind es die Haupt- und Nebenmerkmale (Tabelle 2), die zur sicheren oder möglichen Diagnose TSC führen und in der Regel eine Mutationssuche nach sich ziehen ${ }^{15)}$. Ein negativer Mutationsnachweis schliesst eine TSC nicht aus. Heutzutage wird nicht selten bereits pränatal oder neonatal die Verdachtsdiagnose TSC aufgrund multipler kardialer Rhabdomyome gestellt, die im fetalen Ultraschall entdeckt werden. Früher war es meist im Säuglings- und Kleinkindalter der Fall, wenn die Kombination einer Epilepsie (häufig infantile Spasmen) mit weissen Hautflecken gefunden wurde. Noch heute sind die verschiedenen Hautmanifestationen (Angiofibrome im Gesichtsbereich, fibröse Stirnplaque oder Shagreen Patch) pathognomonisch und sollten an die TSC den-

Der Nachweis einer pathogenen TSC1 oder TSC2 Mutation aus nicht betroffenem Gewebe ist ausreichend für die definitive Diagnosestellung

\begin{tabular}{|c|c|}
\hline Hauptkriterien & Nebenkriterien \\
\hline$\geq 3$ hypomelatonische Flecken & Konfettiflecken der Haut \\
\hline$\geq 3$ Angiofibrome oder Stirnplaque & >3 Zahnschmelzdefekte \\
\hline$\geq 2$ unguale Fibrome & $\geq 2$ Intraorale Fibrome \\
\hline Shagreen Patch & Unpigmentierter Fleck der Retina \\
\hline Hamartome der Retina & Multiple Nierenzysten \\
\hline $\begin{array}{l}\geq 3 \text { kortikale Dysplasien (Tuber, Migrationslinien } \\
\text { der weissen Substanz) }\end{array}$ & Nichtrenale Hamartome \\
\hline Subependymale Gliaknoten & \\
\hline Riesenzellastrozytom & \\
\hline Kardiale Rhabdomyome & \\
\hline Lymphangioleiomyomatose der Lunge (LAM)* & \\
\hline \multicolumn{2}{|l|}{$\geq 2$ Angiomyolipome der Niere* } \\
\hline $\begin{array}{l}\text { Definitive Diagnose } \\
2 \text { Hauptkriterien oder } 1 \text { Hauptkriterium } \\
\text { und } \geq 2 \text { Nebenkriterien }\end{array}$ & $\begin{array}{l}\text { Mögliche Diagnose } \\
1 \text { Hauptkriterium oder } \geq 2 \text { Nebenkriterien }\end{array}$ \\
\hline
\end{tabular}

* Die Kombination LAM und Angiomyolipome ohne andere Zeichen erfüllt nicht die Anforderungen für eine definitive Diagnose. 


\section{Fortbildung}

ken lassen und zur Überweisung an ein spezialisiertes Zentrum führen (Abbildung 2). Ein multidisziplinäres Team ist angesichts der sehr variablen Organbeteiligung erforderlich. Neben den dermatologischen Befunden sind es Manifestationen im ZNS (Epilepsie, Entwicklungsstörung, Riesenzellastrozytom, psychiatrische Störungen), Herz (Rhabdomyome), Nieren (Angiomyolipome, Zysten), Lungen (Lymphangioleiomyomatose LAM), Augen (retinale Hamartome), Zähne, Knochen und seltener auch in anderen Bauchorganen, die lebenslang kontrolliert und behandelt werden müssen. Ca. 20\% der Patienten, meist Kinder oder Jugendliche, entwickeln ein Riesenzellastrozytom im Bereich des Foramen Monroi, welches bei progredientem Wachstum zu einem obstruktiven $\mathrm{Hy}$ drocephalus führen kann ${ }^{16}$ ). Rund $80 \%$ der Patienten leiden an Angiomyolipomen, die bei zunehmendem Wachstum mit einer Blutung akut symptomatisch werden können und langfristig zur Niereninsuffizienz führen ${ }^{17)}$. Für die Überwachung und interdisziplinäre Behandlung betroffener TSC Patienten existieren international breit abgestützte Empfehlungen ${ }^{18)}$.

\section{Behandllung}

In den letzten Jahren konnte durch das Verständnis der molekularbiologischen Vorgänge an einer geziel- ten Therapie geforscht werden, die nicht nur bestehende Symptome lindert, sondern nach Möglichkeit deren Entstehung verhindern soll. Rapamycin (Sirolimus) und das Analogon Everolimus hemmen die mTORC1 Aktivität und führen dadurch zur Kontrolle von unerwünschten Zellproliferation und Wachstum. In Studien, EXIST-1 ${ }^{19)}$ und EXIST-20), wurde gezeigt, dass mit Everolimus Riesenzellastrozytome des Hirns und Angiomyolipome der Nieren kleiner wurden und sich die Lungenfunktion bei LAM stabilisierte. So zeigten mindestens die Hälfte der Patienten eine Volumenreduktion ihres Riesenzellastrozytoms von $>50 \%$. Erwähnenswert ist allerdings die Tatsache, dass nach Absetzen des mTOR-Inhibitors das Tumorwachstum wieder beginnt und darum eine Langzeitbehandlung, eventuell sogar lebenslang, nötig ist. Dementsprechend ist auch eine neurochirurgische Resektion eine zu diskutierende Option und die Therapieentscheidung ist in den Gesamtkontext, unter Berücksichtigung anderer TSC assoziierten Organmanifestationen, zu stellen.

Ca. 85\% der TSC Patienten leiden an einer Epilepsie, die in der Mehrheit pharmakoresistent ist ${ }^{211}$. In der EXIST-3 Studie ${ }^{22)}$ wurde an mehrheitlich pädiatrischen Patienten gezeigt, dass mit Everolimus auch pharma-

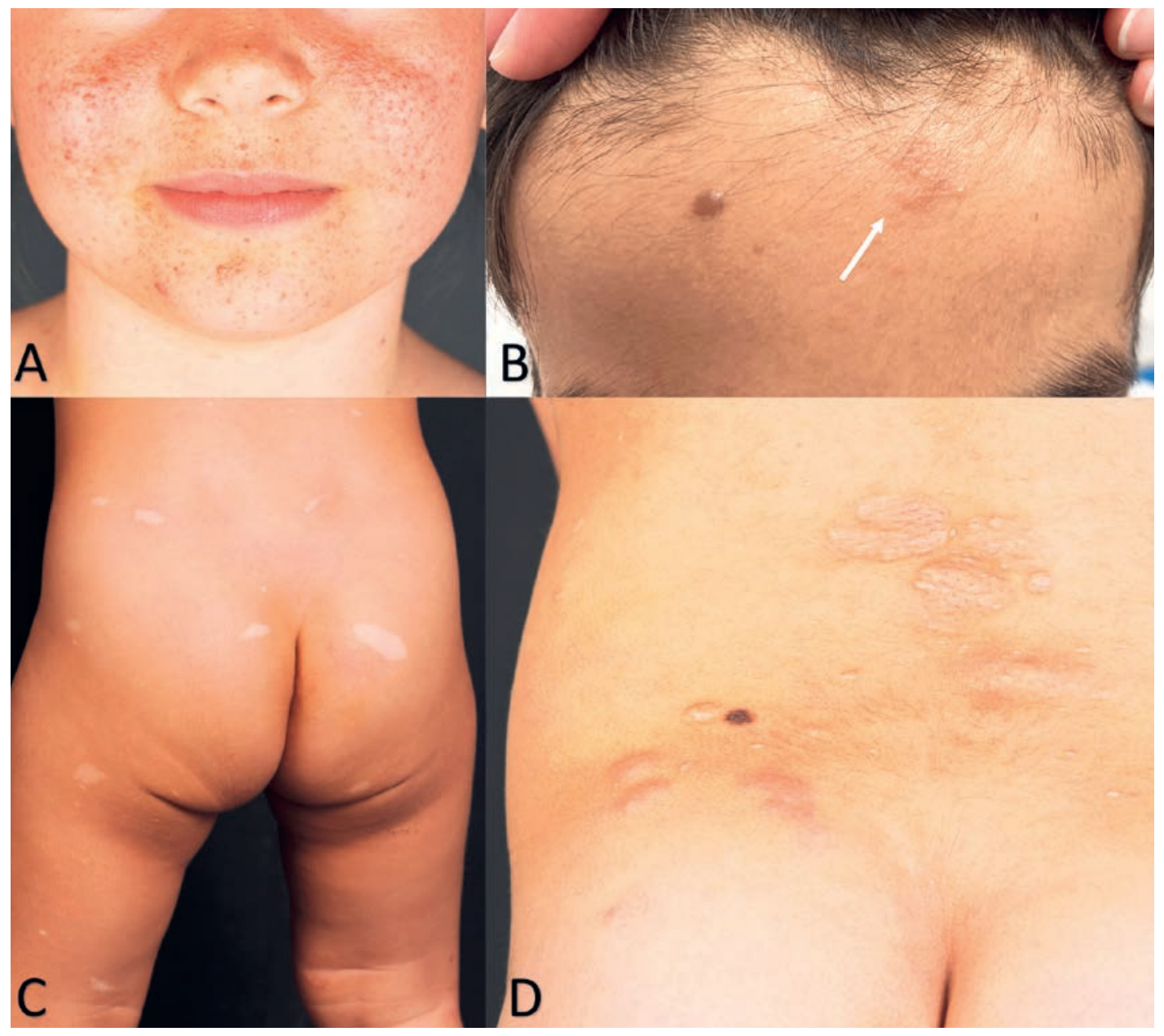

Abbildung 2: Fotos bei Tuberöser Sklerose

A Angiofibrome an Wangen, Nase und Kinn

$B$ Fibröse Stirnplaque links

C Multiple hypomelanotische Flecken

D Shagreen Patch lumbal 


\section{Fortbildung}

koresistente Epilepsiepatienten bezüglich ihrer Anfälle eine Verbesserung erzielen konnten. Der Anteil Patienten mit > 50\% Reduktion der Anfallsfrequenz lag bei $40 \%$ in der Gruppe mit hochdosiertem Everolimus gegenüber $15.1 \%$ in der Placebogruppe. Das Präparat wird nebst den bereits genannten Indikationen bei TSC Patienten auch für weitere TSC assoziierte Krankheitsmanifestationen erfolgreich eingesetzt: Schon im Neugeborenenalter bei grossen Rhabdomyomen des Herzens, um eine kardiochirurgische Intervention abzuwenden und im Kindesalter topisch auf die von Angiofibromen betroffene Gesichtshaut. Zusammengefasst ist es das Ziel der Behandlung mit einem mTOR-Inhibitor, die Grösse und Anzahl der Tumoren bei TSC Patienten zu verringern oder zumindest zu stabilisieren. Eine neue Multizenterstudie ist geplant, um zu untersuchen, ob die prophylaktische Gabe von Sirolimus (Behandlungsbeginn vor dem Alter von 4 Monaten) eine Reduktion neuropsychologischer Defizite zur Folge hat.

\section{Erkrankungen durch genetische Mosaike}

Verschiedene Entwicklungsstörungen und neurokutane Erkrankungen sind durch Neumutationen verursacht, die erst nach der Befruchtung (postzygotisch) auftreten. Man spricht dann auch von sogenannt «somatischen» Mutationen. Solche Mutationen haben zur Folge, dass der Organismus aus genetisch unterschiedlichen Zellpopulationen besteht ${ }^{24}$ ). Obschon zahlreiche Mosaikerkrankungen seit vielen Jahren als klinische Entitäten bekannt waren, wurde deren zugrunde liegende genetische Ursache erst in den letzten Jahren mit Hilfe der Hochdurchsatzsequenzierung (Next Generation Sequencing, NGS) entdeckt. Ein gutes Beispiel dafür ist das sporadisch auftretende Sturge-Weber-Syndrom, das wie eingangs erwähnt zu den ersten beschriebenen neurokutanen Syndromen gehörte, bei dem aber erst 2013 die dafür ursächliche somatische Mutation im GNAQ Gen in betroffenem Gewebe nachgewiesen wurde ${ }^{25}$. Damit wurde eine seit langem bestehende Hypothese, die der Mosaikerkrankung, bestätigt. Für die genetische Beratung der Eltern verändert der Nachweis einer somatischen Mutation die Ausgangslage erheblich, besteht doch in diesem Fall bei weiteren Kindern kein erhöhtes Wiederholungsrisiko für die gleiche Erkrankung.

Vaskuläre Fehlbildungen können assoziiert sein mit einer breiten Palette von kutanen, neurologischen oder muskuloskelettalen Befunden. Historisch sind zahlreiche Syndrome mit Eigennamen (wie Klippel-Trénaunay-Syndrom) oder Akronymen (wie CLOVES Syndrom) und zum Teil überlappenden Phänotypen beschrieben worden. Die Entdeckung somatischer aktivierender Mutationen im PIK3CA Gen hat dann die zugrundeliegende Ursache verschiedener klinisch definierter Phänotypen geklärt. Für diese Syndrome mit der Kombination von vaskulären Fehlbildungen und segmentalen/fokalen Hypertrophien wurde der neue Überbegriff PROS für PIK3CA-Related Overgrowth Spectrum geschaffen ${ }^{26)}$. Der Mutationsnachweis ist allerdings wegen des Mosaiks nicht einfach und es muss mit modernen Technologien (NGS) primär in betroffenem Gewebe (häufig in Fibroblasten) und nicht im Blut gesucht werden. Ein fehlender Mutationsnachweis schliesst die Diagnose eines PROS nicht aus.

\section{Megalencephaly-capillary Malformation}

\section{Syndrom (MCAP)}

Das MCAP ist ein Vertreter dieser PROS Gruppe, welches uns im Alltag der neurokutanen Sprechstunde wiederholt begegnet und möglicherweise unterdiagnostiziert ist. Die Betroffenen haben einen disproportional grossen bis sehr grossen Kopf und kapilläre Fehlbildungen auf der Haut, gehäuft ein asymmetrisches Wachstum von Gliedmassen und eine Bindegewebsauffälligkeit mit Laxizität. Relativ charakteristisch ist eine faziale kapilläre Malformation der Gesichtsmittellinie (Abbildung 3). Ein Teil der Patienten hat zusätzliche ZNS-Fehlbildungen wie Polymikrogyrien oder eine Hemimegalenzephalie mit dem Risiko von Entwicklungsstörungen und Epilepsien. Das sehr rasche Kopfwachstum in den ersten Lebensjahren mit den typischerweise relativ weiten Seitenventrikeln in der Bildgebung muss sorgfältig, auch mittels MRI, kontrolliert und interpretiert werden, um einerseits nicht unnötig einen Shunt zu implantieren, und andererseits die seltene Komplikation einer Kleinhirnherniation mit Entwicklung eines obstruktiven Hydrozephalus, nicht zu verpassen. Kopfumfänge von $55-58 \mathrm{~cm}$ im Alter von zwei Jahren sind keine Seltenheit. Auch beim MCAP ist eine somatische PIK3CA Mutation zugrundeliegend, wobei hier gelegentlich der Mutationsnachweis auch im Blut und nicht nur in betroffener Haut gelingt. Der Mutationsnachweis ist wichtig wegen der neuen zielgerichteten Therapiemöglichkeiten. Alpelisib (BYL719), ein PIK3CA-Inhibitor, hat in einer Studie mit PROS Spektrum Patienten ${ }^{27)}$ zu bemerkenswerten Verbesserungen geführt: Rückgang der kapillären Malformation und epidermalen Naevi, Verbesserung der Skoliose, Beendigung einer chronischen gastrointestinalen Blutung. Weitere Resultate aus Studien mit Alpelisib und betroffenen Kindern und Jugendlichen werden bald erwartet.

\section{Schlussbemerkung}

Der Pädiaterin und dem Pädiater wird eine wichtige Rolle zuteil im frühen Erkennen der Verdachtsdiagnose aufgrund der klinischen Symptome und dem Zuweisen an ein erfahrenes Zentrum. Die Entwicklung der letzten Jahre mit verbesserten diagnostischen und vor allem therapeutischen Möglichkeiten macht die multidisziplinäre Betreuung dieser Patienten umso wichtiger. Dank: Ich bedanke mich bei Kindern und Eltern für die Erlaubnis zur Publikation der Fotos und bei Lisa Weibel, Martin Theiler und Eugen Boltshauser für die Mithilfe im Bereitstellen von Bildmaterial.

Für das Literaturverzeichnis verweisen wir auf unsere Online Version des Artikels. 


\section{Fortbildung}

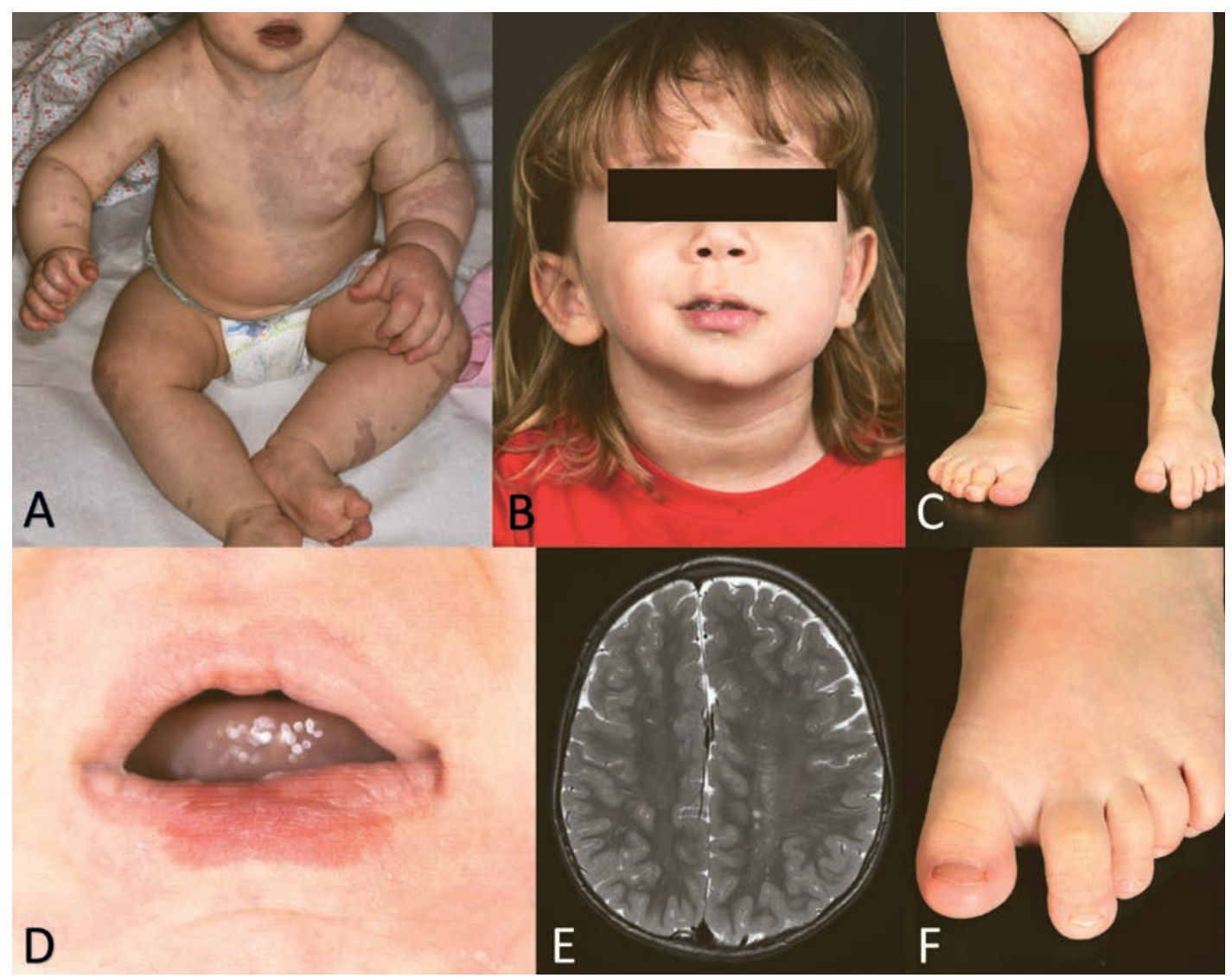

Abbildung 3: Fotos und MRI Bild bei Megalencephaly-capillary Malformation Syndrom

A Ausgedehnte livide kapilläre Malformation sowie Hypertrophie des linken Armes

B Hypertrophie linke Gesichtshälfte

C Asymmetrisches Körperwachstum mit Hypertrophie rechtes Bein

D Kapilläre Malformation Unterlippe

E Hemimegalenzephalie links passend zur Gesichtsasymmetrie Bild B

F Makrodaktylie der 2. Zehe

\section{Autorin}

Dr. med. Sandra Tölle, Abteilung Neuropädiatrie, Universitäts-Kinderspital Zürich - Eleonorenstiftung, Zürich

Die Autorin hat keine finanziellen oder persönlichen Verbindungen im Zusammenhang

mit diesem Beitrag deklariert. 